\title{
Lattice Boltzmann Simulation of the Flow over Wavy Surface
}

\author{
W. P. Shi*, L. X. Ding, H. C. Zheng \\ College of Mathematics, Jilin University, Changchun 130012, China \\ Email: shiwp@jlu.edu.cn
}

\begin{abstract}
Skin friction drag has been an issue of great interest. Any drag reduction of air flight or underwater travel can lead to significant energy savings. This increase in interest is associated with demands for better performance and less fuel consumption of vehicles. Because skin friction drag is the interaction between a solid surface and fluid, the magnitude of skin friction drag depends on the properties of both the solid and the fluid. Along the solid surface, a boundary layer of low energy flow is generated. The magnitude of the skin friction depends on the state of this flow. Non-planar walls are being investigated. Among them some kinds of rough surfaces are one of the candidate surfaces for drag reduction.

Lattice Boltzmann method (LBM) is a tool of computational fluid dynamics, which is based on the microscope dynamics of fluid. Because the method is explicit, easy to implement and parallelize, it has been developed into a promising numerical scheme for simulating fluid flows in various systems in recent years.

This paper deals with the simulation of the two-dimensional flow over wavy surfaces and the investigation of drag reduction for the laminar flow over wavy surface. The flows over the different concave surfaces are simulated based on the lattice Boltzmann method. The fluid is incompressible and the inlet velocity is uniform and parallel to the plate. The boundary treatment of second order accuracy and the formula for evaluation the forces acting on the curved surface are constructed by the momentum- exchange method in the present simulation. The numerical results show that for the laminar flow the drag of the triangular concave surface is less than that of the sinusoidal surface. The momentum-exchange method is recommended for force evaluation on curved boundaries for its simplicity, accuracy, and robustness. The lattice Boltzmann method is reliable, accurate and effective for this kind of calculation.
\end{abstract}

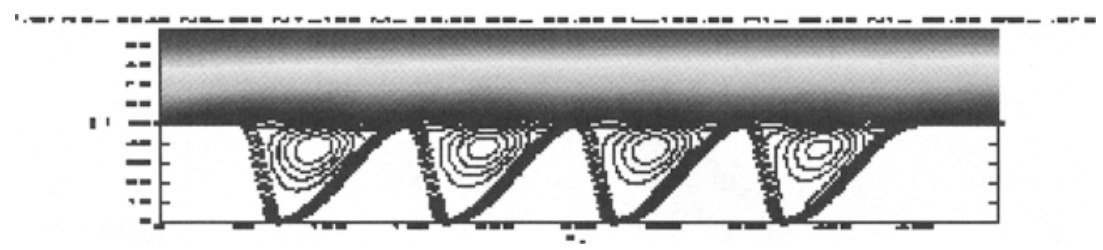

Figure. The streamlines of flow over sinusoidal surface, $R e=2000$, drag coefficient $C_{D}=0.072$

\section{REFERENCES}

1. Shi W, Hu S, Yan G. A lattice Boltzmann equation method for the shallow water wave equations. ACTA Mechanica Sinica, 1997; 29: 525-529 (in Chinese)

2. Mei R, Luo L, Shyy W. An accurate curved boundary treatment in the lattice Boltzmann method. Journal of Computational Physics, 1999; 155: 307-330

3. Mei R, Yu D, Shyy W, Luo L-S. Force evaluation in the lattice Boltzmann method involving curved geometry. Physical Review E, 2002; 65: 041203/1-041203/14 